\title{
Field-Line Localized Destabilization of Ballooning Modes in Three-Dimensional Tokamaks
}

\author{
M. Willensdorfer, ${ }^{1, *}$ T. B. Cote, ${ }^{2, \dagger}$ C. C. Hegna, ${ }^{2}$ W. Suttrop,${ }^{1}$ H. Zohm,,${ }^{1}$ M. Dunne, ${ }^{1}$ E. Strumberger, ${ }^{1}$ \\ G. Birkenmeier, ${ }^{1,3}$ S. S. Denk, ${ }^{1,3}$ F. Mink, ${ }^{1}$ B. Vanovac, ${ }^{4}$ L. C. Luhmann ${ }^{5}$ and the ASDEX Upgrade Team ${ }^{1}$ \\ ${ }^{1}$ Max Planck Institute for Plasma Physics, 85748 Garching, Germany \\ ${ }^{2}$ University of Wisconsin-Madison, Madison, Wisconsin 53706, USA \\ ${ }^{3}$ Physik-Department E28, Technische Universität München, 85748 Garching, Germany \\ ${ }^{4}$ FOM-Institute DIFFER, Dutch Institute for Fundamental Energy Research, 5612 AJ Eindhoven, Netherlands \\ ${ }^{5}$ University of California at Davis, Davis, California 95616, USA
}

(Received 27 March 2017; published 25 August 2017)

\begin{abstract}
Field-line localized ballooning modes have been observed at the edge of high confinement mode plasmas in ASDEX Upgrade with rotating 3D perturbations induced by an externally applied $n=2$ error field and during a moderate level of edge localized mode mitigation. The observed ballooning modes are localized to the field lines which experience one of the two zero crossings of the radial flux surface displacement during one rotation period. The localization of the ballooning modes agrees very well with the localization of the largest growth rates from infinite- $n$ ideal ballooning stability calculations using a realistic 3D ideal magnetohydrodynamic equilibrium. This analysis predicts a lower stability with respect to the axisymmetric case. The primary mechanism for the local lower stability is the 3D distortion of the local magnetic shear.
\end{abstract}

DOI: 10.1103/PhysRevLett.119.085002

Introduction.-In order to mitigate the possible harmful heat load from edge localized modes (ELMs) in the high confinement mode ( $H$ mode) in future fusion devices, it is necessary to suppress or to mitigate the ELMs. The application of magnetic perturbations (MPs) enables mitigation of ELMs and under certain circumstances even suppression. This method has the side effect of a reduced plasma density at low collisionality $\nu^{\star}$ and reduced pedestal plasma pressure, the so-called density "pump out" [1].

In recent years, there is growing evidence that stable ideal kink modes can amplify the externally applied MP field [2,3], which plays a key role in ELM mitigation [4] and ELM suppression at low $\nu^{\star}[5,6]$. These magnetohydrodynamic (MHD) modes are driven by the edge pressure gradient and/or the associated bootstrap current. Comparative studies in combination with MHD modeling indicate that the highest ELM frequency, the strongest density pump out, and the strongest accompanying reduction in the edge pressure gradient are correlated with the strongest coupling between stable ideal kink modes $(m>n q)$ and resonant components of rational surfaces ( $m=n q$ ), where $m$ and $n$ are the poloidal and toroidal mode numbers, respectively, and $q$ is the safety factor [7-10]. The resulting three-dimensional (3D) boundary distortion can be many times larger than expected solely from the MP of the vacuum field $[11,12]$. It has been argued

Published by the American Physical Society under the terms of the Creative Commons Attribution 4.0 International license. Further distribution of this work must maintain attribution to the author(s) and the published article's title, journal citation, and DOI. that the MPs modify the peeling-ballooning mode (PBM) stability by a change of the edge bootstrap current due to the density pump out [13] or equilibrium currents around rational surfaces [14]. To approximate changes in the finite- $n$ PBM stability [13], the local infinite- $n$ ideal MHD ballooning theory is often used as an estimate.

The infinite- $n$ ballooning theory in 3D MHD geometry has been extensively studied for stellarator configurations, e.g., Refs. $[15,16]$. The first theoretical attempts to apply it to a 3D tokamak geometry induced by external MPs were done in Ref. [17]. It was proposed that the 3D modulations of the local magnetic shear associated with the presence of near-resonant Pfirsch-Schlüter currents perturb the ballooning stability boundary. This can lead to enhanced growth rates on local field lines.

In this Letter, we present novel first measurements, which demonstrate field-line localized destabilization of ideal MHD ballooning modes in the presence of an edge-perturbed 3D MHD tokamak equilibrium. The measured localization of the ballooning modes in the 3D geometry combined with infinite- $n$ ballooning calculations supports the proposed importance of the changes in the local magnetic shear.

Experimental setup.-The presented example is from a series of ASDEX Upgrade experiments to measure the displacement by combining rigidly rotating MP fields with the toroidal mode number $n=2$ and toroidally localized diagnostics [18]. In this work, we primarily use electron cyclotron emission (ECE) diagnostics, which deliver the electron temperature $\left(T_{e}\right)$ around the low field side (LFS) midplane from the measured radiation temperature $\left(T_{\text {rad }}\right)$ [19]. To have the best coverage at the edge for profile-ECE and ECE-imaging (ECE-I) [20] measurements, we set a 


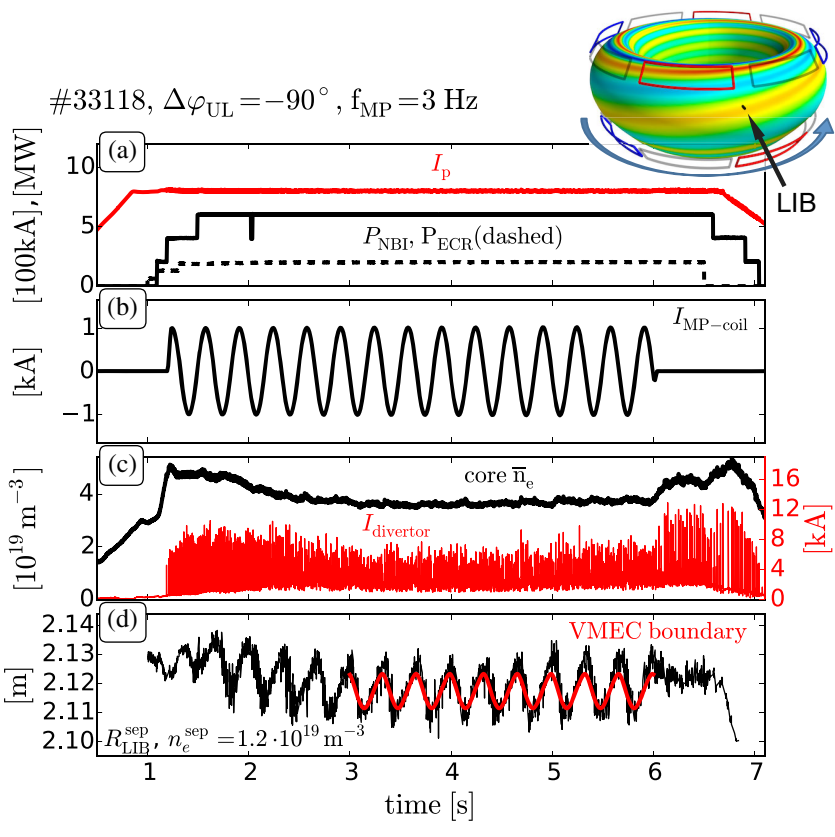

FIG. 1. Time traces from a discharge with a rigidly rotating MP field resulting in a rotation of the $\xi_{r}$. The inset shows corresponding $\xi_{r}$ from VMEC (red outwards, green zero, blue inwards at the boundary) and coil configuration at $4 \mathrm{~s}$. (a) Plasma current, external ECR, and NBI heating power, (b) supply current of one coil, (c) core $\overline{n_{e}}$ and divertor current $\left(I_{\text {divertor }}\right)$, and (d) separatrix movements along the LIB from measured pre-ELM $n_{e}$ profiles and the VMEC boundary. Good agreement is found.

toroidal field $\left(\left|B_{T}\right|\right)$ of around $2.5 \mathrm{~T}$. Further global parameters are a plasma current $I_{P}$ of $800 \mathrm{kA}$, an edge safety factor $q_{95}$ of 5.3, and a core electron density $\left(n_{e}\right)$ of $4.3 \times 10^{19} \mathrm{~m}^{-3}$. The applied neutral beam injection (NBI) and electron cyclotron resonance (ECR) heating are approximately 6 and $2 \mathrm{MW}$, respectively [see Fig. 1(a)]. A slow $\left(f_{\mathrm{MP}}=3 \mathrm{~Hz}\right)$ rotating MP field is applied. The MPcoil supply current shown in Fig. 1(b) illustrates the timing. The rotation is in the positive toroidal direction using a fixed differential phase angle $\Delta \varphi_{\mathrm{UL}}$ of around $-90^{\circ}$ between the MP field from the upper and lower coil set (see the cartoon in the right top corner of Fig. 1). Because of eight coils in each row and a low intrinsic error field, the dominant $n=2$ spectrum varies little during the rotation as seen by small core $n_{e}$ perturbations $(<5 \%)$.

The applied MP-field configuration $\left(\Delta \varphi_{\mathrm{UL}} \approx-90^{\circ}\right)$ is suitable to excite stable ideal kink modes at the edge [9], which leads to a moderate level of density pump out and a reduction of the ELM size. This is best seen in Fig. 1(c) at $6 \mathrm{~s}$ during the switch off of the MP field, where a recovery of the core line averaged electron density $\overline{n_{e}}$ is shown (black) and an increase of the particle flux towards the divertor during ELM crashes. The latter one is indicated by thermoelectric currents into the divertor tile [21] measured by the voltage drop across a shunt resistor [red in Fig. 1(c)].

The rigidly rotating MP field causes a rotation of the radial displacement $\left(\xi_{r}\right.$, normal to the axisymmetric flux surface) at the boundary, which is seen as a modulation in edge profile diagnostics. To track $\xi_{r}$ at the boundary, we use the $n_{e}$ profiles from the lithium beam (LIB) diagnostic [22] assuming a constant separatrix density [Fig. 1(d)].

To model $\xi_{r}$, we employ the 3D ideal MHD equilibrium code VMEC [23]. If no strong resistive MHD modes are active, VMEC is able to predict $\xi_{r}$ at the edge $[11,18]$. For details about its setup for ASDEX Upgrade discharges and the accuracy of VMEC in the presence of rational surfaces, we refer to Refs. $[18,19,24]$ and Refs. $[25,26]$, respectively. For the given configuration, VMEC predicts a maximum $\xi_{r}$ of $\pm 11 \mathrm{~mm}$ at the plasma top and $\pm 6 \mathrm{~mm}$ around the LFS midplane (visualized in the inset in Fig. 1). To underline the agreement of the calculated $\xi_{r}$ with experiments, the predicted boundary corrugation from the 3D VMEC equilibrium along the lines of sight (LOS) of the LIB is added in Fig. 1(d). This equilibrium is calculated once at $4 \mathrm{~s}$, and the corrugation is then mapped onto the time base according to the phase of the MP-field rotation [19]. Good agreement is found, and the amplitudes differ by not more than $1.3 \mathrm{~mm}$.

Observations.-During rigid rotation experiments, we observe an instability occurring only at certain toroidal phases. Figure 2 shows time traces of the measured relative

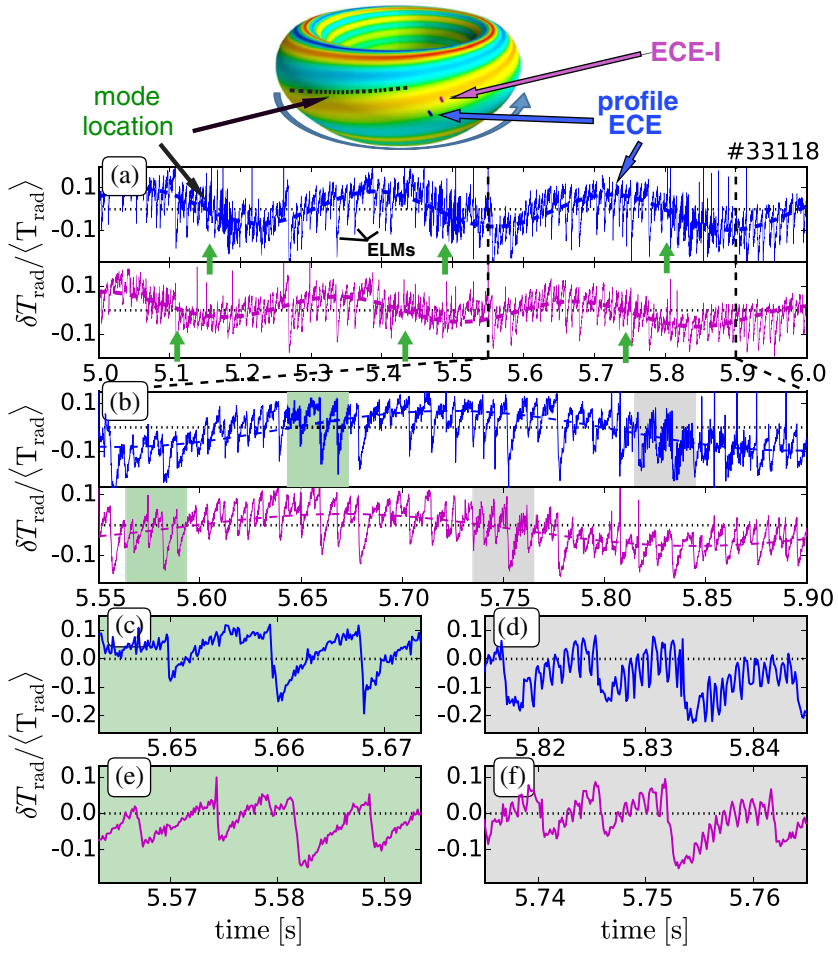

FIG. 2. Time traces from profile ECE (blue) and ECE-I (purple) channels. Horizontal dotted lines indicate the zero line. Measuring principle and LOS positions are indicated at the top. (a) A $3 \mathrm{~Hz}$ modulation due to the rotating $\xi_{r}$; (b) a MHD mode with $f \approx 1 \mathrm{kHz}$ is seen when one specific $\xi_{r} \approx 0$ [indicated by green arrows in (a)] passes the diagnostics. The mode appears in between ELM crashes and at only one $\xi_{r} \approx 0(\mathrm{~d}),(\mathrm{f})$, whereas at the other times not, e.g., (c),(e). Please note the different time ranges between (c)-(f) illustrated by shaded areas in (b). 
amplitude ( $\delta T_{\mathrm{rad}} /\left\langle T_{\mathrm{rad}}\right\rangle$ with $\left.\delta T_{\mathrm{rad}}=T_{\mathrm{rad}}-\left\langle T_{\mathrm{rad}}\right\rangle\right)$, where $\left\langle T_{\mathrm{rad}}\right\rangle$ is averaged over the three periods, from one profile ECE (blue) and one ECE-I (magenta) channel probing the steep gradient region near the pedestal top. These channels are continuously optically thick, so we can assume $T_{e} \approx T_{\text {rad }}$. Three wave periods and multiple ELM crashes are seen in Fig. 2(a). ELM crashes are visible as sudden drops, and the modulation from the rotating $\xi_{r}$ is emphasized by fits of sinusoidal functions (dashed colored lines) using only pre-ELM data points. Because of the poloidally as well as toroidally separated measurement positions and the alignment of $\xi_{r}$ (see the cartoon in Fig. 2), the two diagnostics exhibit a relative phase shift of around $\pi / 2$.

High-frequency modes $(f \approx 1 \mathrm{kHz})$ are measured by the diagnostics, when field lines with specific 3D geometry pass their LOS (green arrows). To enhance their visibility, Fig. 2(b) magnifies one period. It is seen that these modes develop at different times in the profile ECE and ECE-I channels but at the same toroidal phase with respect to the $3 \mathrm{~Hz}$ modulation [gray shaded areas in Fig. 2(b)]. They appear in between ELMs, and during one period they occur only once when the modulation crosses zero (dotted line) from positive to negative values [Figs. 2(d) and 2(f)]. At other times, such pronounced oscillations are not seen, e.g., Figs. 2(c) and 2(e). The amplitude of the mode appears to be smaller in the ECE-I in comparison to the profile ECE channel [Figs. 2(d) and 2(f)]. This is simply because of the twice as large observation volume of the ECE-I resulting in a smearing of the amplitude.

To demonstrate the properties of an ideal MHD instability, Fig. 3 shows $T_{e}$ profiles during the ELM recovery

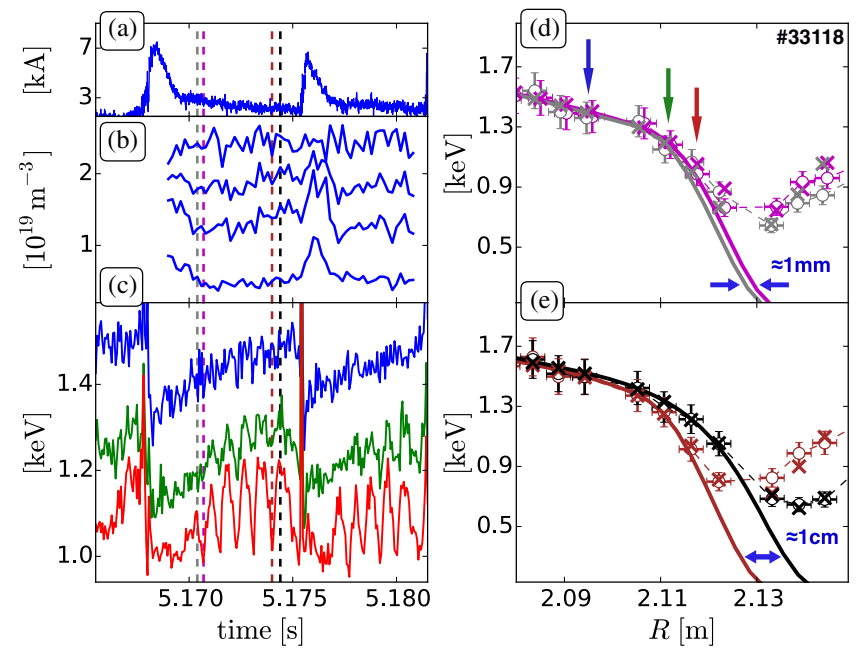

FIG. 3. (a) Divertor currents, (b) edge $n_{e}$ from LIB, and (c) $T_{\text {rad }}$ in the gradient region. (d),(e) $T_{e}$ profiles (solid lines) and the corresponding modeled $(\times)$ and measured $T_{\text {rad }}$ (circles with bars). The timing of the profiles are indicated by colored vertical lines in (a)-(c). The first pair of vertical lines (gray and purple) corresponds to (d) and the second (brown and black) to (e). Arrows in (d) indicate ECE channels used in (c). The boundary deformation induced by the $1 \mathrm{kHz}$ mode increases during the pedestal recovery. determined from forward modeling of the measured $T_{\text {rad }}$ profiles [27]. After the ELM crash, the edge pressure profile recovers as indicated by a slight steepening of $n_{e}$ in the edge gradient region [Fig. 3(b)] and an increase of $T_{e}$ at the pedestal top [blue in Fig. 3(c)]. Simultaneously, the boundary deformation due to the high-frequency mode increases from less than $1 \mathrm{~mm}$ to around $1 \mathrm{~cm}$ [Figs. 3(d) and 3(e)], which is suggestive for a pressure-gradientdriven instability. The $1 \mathrm{kHz}$ mode causes clear radial displacements in the pedestal. There is no indication of a magnetic island such as profile flattening at a rational surface or the $T_{e}$ perturbations being in antiphase on both sides of a rational surface.

The high-frequency modes are also seen in other profile diagnostics around the LFS midplane like LIB. They are also measured at the LFS by soft x-ray, diode bolometers, and weakly in magnetic probes, but these diagnostics do not detect these modes at the high field side. This gives us confidence that we are dealing with ideal MHD ballooning modes. According to ECE-I measurements, the mode rotates toroidally into the opposite direction of the MP-field rotation, so clockwise and poloidally into the electron diamagnetic direction as expected from ballooning modes [28].

These ballooning modes are also observed in other discharges from this experimental series. Figures 4(a)-4(c) show profile ECE measurements from rigid rotation experiments using the identical plasma configuration but with $f_{\mathrm{MP}}=2 \mathrm{~Hz}$ in the positive toroidal direction and various $\Delta \varphi_{\mathrm{UL}}$ 's. Although these experiments have different applied poloidal mode spectra set by $\Delta \varphi_{\mathrm{UL}}$ [18], two features are

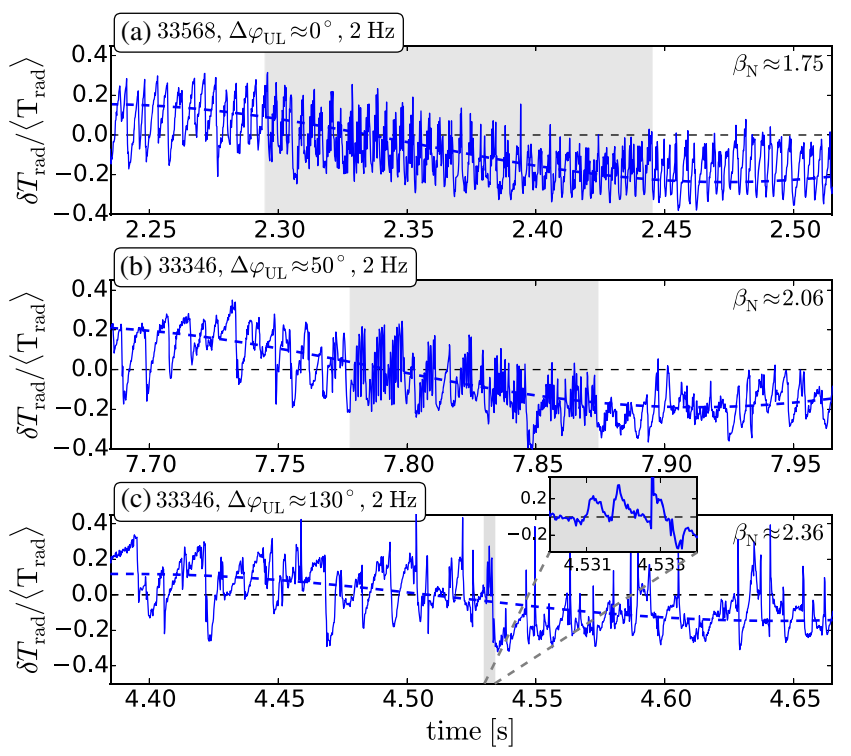

FIG. 4. (a)-(c) The same time traces from the profile ECE as in Fig. 2 using $f_{\mathrm{MP}}=2 \mathrm{~Hz}$ and various $\Delta \varphi_{\mathrm{UL}}$ 's. The appearance of the ballooning mode is indicated by the gray shaded area. The inset in (c) is the enlargement of the gray shaded area, which shows two periods of the mode. $\beta_{N}$ values averaged over one MP-rotation period are in the right top corners. 
noticeable: (i) The ballooning modes appear primarily around the same field lines, which experience the zero crossing of $\xi_{r}$ from positive to negative values when the MP field is rotated in the positive toroidal direction. (ii) The duration of the observation window differs with the coil configurations, which indicates that the poloidally and the toroidally expansion of the ballooning modes vary. A larger expansion is seen for applied coil configurations [e.g., $\Delta \varphi_{\mathrm{UL}} \approx 0^{\circ}$ in Fig. 4(a)], which favor the excitation of the stable ideal kink modes resulting in larger $\xi_{r}$ (illustrated by dashed lines), increased ELM frequency, and lower pressure indicated by the normalized beta $\beta_{N}$ in Fig. 4 . The ballooning modes are barely, if at all, seen in the case in which $\xi_{r}$ is small and $\beta_{N}$ largest [Fig. 4(c)]. This indicates that these stable ideal kink modes are correlated not only with the ELM behavior and the changes in pressure but also with the appearance of the ballooning modes. The observation of the ballooning modes is not limited to the presented plasma configuration. For example, they are also seen in experiments at $\left|B_{T}\right| \approx 2.0 \mathrm{~T}$ with dominant ion cyclotron resonance heating and $q_{95} \approx 4.13$.

In summary, we observe rotating ballooning modes, which are dominantly destabilized around a certain field line in the 3D flux surface geometry. These ballooning modes do not appear at the maximum $\xi_{r}$, where the local expansion of the flux surfaces causes the largest changes to the normal curvature and the smallest peak pressure gradients in real space $\partial p / \partial r$. They are not dominantly destabilized at the minimum $\xi_{r}$, where the local compression of the flux surfaces leads to the largest pressure gradients. This is different from the observed ballooning modes in the presence of internal kink modes in the core. In these cases, the largest gradients come with the largest displacements, which then locally destabilize the ballooning modes in the core [29]. In the presence of perturbed flux surfaces from externally applied MPs, the modes are dominantly destabilized, where $\xi_{r}$ is zero as predicted by Ref. [17]. Even more interestingly, they primarily appear around one specific zero crossing, although there are two in each period. The reason for this will be elucidated in the following.

Stability analysis.-Motivated by the experimental observations, we extended the stability analysis from Ref. [17] using the 3D equilibrium from VMEC introduced previously (Fig. 1) and its $n=0$ solution for the twodimensional (2D) case. The addition of 3D magnetic perturbations causes the local plasma stability to vary on different magnetic field lines in the steep gradient region of the pressure profile. Applying the ideal ballooning theory allows us to solve for the high- $n$, local plasma stability on individual field lines. The calculated growth rates $\gamma$ (color scaling) in the pedestal are shown in Fig. 5 as a function of the field line label $\alpha=q \Theta^{\star}-\phi$ with the straight field-line coordinates $\left(\phi, \Theta^{\star}\right)$ [23] versus the square root of the normalized toroidal flux $\left(\rho_{\text {tor }}\right)$ for one $n=2$ period. In comparison to the axisymmetric $n=0$ case [Fig. 5(a)], the growth rates of the perturbed
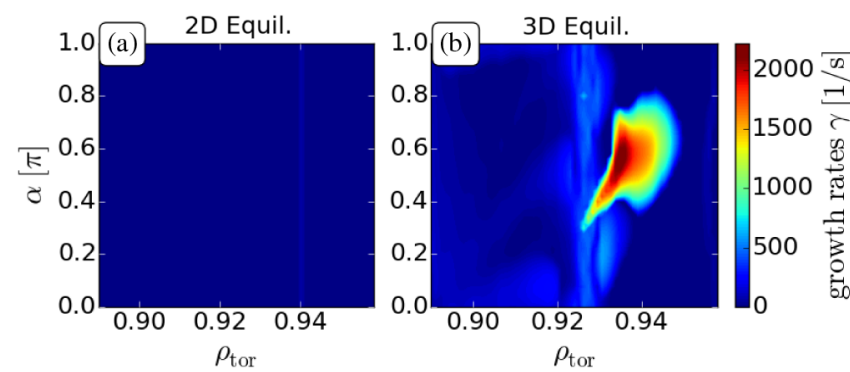

FIG. 5. Growth rates $\gamma$ on the field line label $\alpha$ versus $\rho_{\text {tor }}$ for (a) axisymmetric $2 \mathrm{D}$ and (b) perturbed $3 \mathrm{D}$ equilibrium. The $3 \mathrm{D}$ case has locally enhanced $\gamma$ and is most unstable around $\alpha=0.6 \pi$.

equilibrium are enhanced [Fig. 5(b)], which indicates a lower stability in the 3D case with respect to the axisymmetric case. The growth rates are largest around the field line $\alpha=0.6 \pi$.

To identify the position of the most unstable field line $\alpha=0.6 \pi$ in the 3D geometry, Fig. 6(a) shows $\xi_{r}$ on the unfolded axisymmetric flux surface together with this field line. It is located at the same zero crossing as the measured localization of the ballooning mode in Fig. 2. To elucidate the reason for this specific field line to be unstable, Fig. 6(b) shows the corresponding local magnetic shear [defined by $s=\hat{b} \times \hat{n} \cdot \boldsymbol{\nabla} \times(\hat{b} \times \hat{n})$, where $\hat{b}$ and $\hat{n}$ are the unit magnetic field and normal components to the magnetic surface, respectively [30]] versus $\Theta^{\star}$ for several field lines. $s$ enters the ballooning equation [17] and stabilizes ballooning modes against field-line bending. Thus, the most unstable field line exhibits the lowest absolute values of $s$ in the region, where the mode is primarily driven. This is in the present equilibrium between the LFS midplane and the plasma top [Fig. 6(b)], where the normal curvature is negative and the geodesic curvature is positive [17].
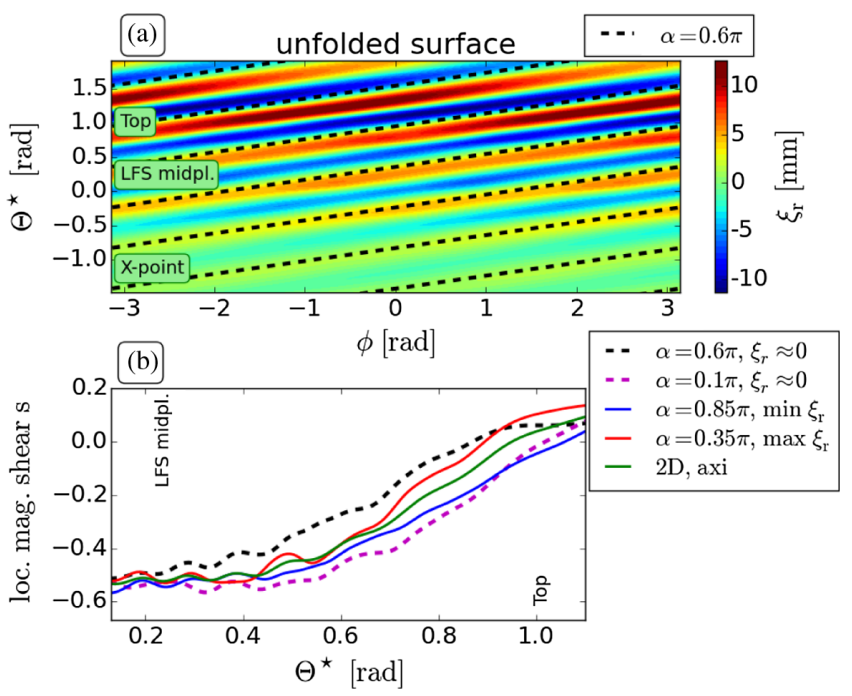

FIG. 6. (a) Unfolded surface at $\rho_{\text {tor }} \approx 0.935$ showing the radial displacement $\xi_{r}$. Positive values are pointing outwards. The dashed line indicates the most unstable field line $\alpha=0.6 \pi$. (b) Local magnetic shear $s$ versus $\Theta^{\star}$ for various field lines. The most unstable field line has the lowest magnitude of $s$. 
Although the changes in $s$ are small, they are enough to significantly vary the growth rates of the ballooning modes as seen in Fig. 5.

The change in local magnetic shear is related to changes in the local parallel current profile and geometric shape of the magnetic surface as described by the identity $s=\mu_{0} J_{\|} / B-2 \tau_{n}$, where the normal torsion is defined by $\tau_{n}=-\hat{n} \cdot(\hat{b} \cdot \boldsymbol{\nabla}) \hat{b} \times \hat{n}[30]$. Near rational surfaces in 3D MHD equilibrium, Pfirsch-Schlüter currents become resonant and hence can produce large distortions in the local magnetic shear [17]. However, the analysis of the equilibrium presented here shows that the 3D-distortion-produced changes in the normal torsion are responsible for the minimization of the local magnetic shear in the vicinity of the field line $\alpha=0.6 \pi$ [31]. The 3D distortions, however, do not produce appreciable changes to field-line curvature, indicating that the dominant reason for ballooning destabilization is the reduction of stabilizing field-line bending.

Discussion.-The presented measurements combined with 3D ideal MHD modeling evidence the importance of the additional 3D geometry on the infinite- $n$ ballooning stability. The localization of the ballooning modes identifies the variation of the local magnetic shear as the dominant mechanism in the additional destabilization. Our observation and analysis suggest that the reduced edge pedestal pressure in $H$-mode plasmas with nonaxisymmetric MPs is due to a modification of the edge stability boundary introduced by the 3D distortion of the local magnetic shear. Furthermore, it could also deliver reasonable explanations for enhanced transport, the density pump out, since the local magnetic shear can also influence further curvature-driven instabilities like kinetic ballooning modes (KBMs) and drift waves (DWs) $[32,33]$. Nevertheless, dedicated studies using 3D stability calculations of, e.g., PBM [34], KBM [35], and DW [32] are needed to pin down their role and the role of the 3D equilibrium in the ELM mitigation and suppression. Finally, we would like to point out that the described mechanism is solely based on single-fluid ideal MHD physics and does not invoke any mode penetration or ergodization [36].

This research was supported by U.S. Department of Energy under Grant No. DE-FG02-86ER53218. This work has been carried out within the framework of the EUROfusion Consortium and has received funding from the Euratom research and training programme 2014-2018 under Grant Agreement No. 633053. The views and opinions expressed herein do not necessarily reflect those of the European Commission.

*matthias.willensdorfer@ipp.mpg.de †tcote@wisc.edu

[1] T. E. Evans et al., Phys. Rev. Lett. 92, 235003 (2004).

[2] Y. Liu, A. Kirk, Y. Gribov, M. P. Gryaznevich, T. C. Hender, and E. Nardon, Nucl. Fusion 51, 083002 (2011).

[3] C. Paz-Soldan et al., Phys. Rev. Lett. 114, 105001 (2015).
[4] A. Kirk et al., Nucl. Fusion 55, 043011 (2015).

[5] R. Nazikian et al., Phys. Rev. Lett. 114, 105002 (2015).

[6] W. Suttrop et al., Plasma Phys. Controlled Fusion 59, 014049 (2017).

[7] C. Paz-Soldan, N. C. Logan, S. R. Haskey, R. Nazikian, E. J. Strait, X. Chen, N. M. Ferraro, J. D. King, B. C. Lyons, and J.-K. Park, Nucl. Fusion 56, 056001 (2016).

[8] D. A. Ryan et al., Plasma Phys. Controlled Fusion 57, 095008 (2015).

[9] Y. Liu et al., Nucl. Fusion 56, 056015 (2016).

[10] F. Orain et al., Nucl. Fusion 57, 022013 (2017).

[11] I. T. Chapman et al., Nucl. Fusion 54, 083006 (2014).

[12] R. A. Moyer, M. A. Van Zeeland, D. M. Orlov, A. Wingen, T. E. Evans, N. M. Ferraro, J. M. Hanson, R. Nazikian, M. R. Wade, and L. Zeng, Nucl. Fusion 52, 123019 (2012).

[13] I. T. Chapman et al., Phys. Plasmas 20, 056101 (2013).

[14] C. J. Ham, I. T. Chapman, J. Simpson, and Y. Suzuki, Plasma Phys. Controlled Fusion 57, 054006 (2015).

[15] J. N. Talmadge and W. A. Cooper, Phys. Plasmas 3, 3713 (1996).

[16] C. C. Hegna and N. Nakajima, Phys. Plasmas 5, 1336 (1998).

[17] T. M. Bird, and C. C. Hegna, Nucl. Fusion 53, 013004 (2013).

[18] M. Willensdorfer et al., Nucl. Fusion 57, 116047 (2017).

[19] M. Willensdorfer et al., Plasma Phys. Controlled Fusion 58, 114004 (2016).

[20] I. G. J. Classen, C. W. Domier, N. C. Luhmann, A. V. Bogomolov, W. Suttrop, J. E. Boom, B. J. Tobias, and A. J. H. Donné, Rev. Sci. Instrum. 85, 11D833 (2014).

[21] G. Pautasso, L. Giannone, O. Gruber, A. Herrmann, M. Maraschek, and K. H. Schuhbeck, Nucl. Fusion 51, 043010 (2011)

[22] M. Willensdorfer, G. Birkenmeier, R. Fischer, F. M. Laggner, E. Wolfrum, G. Veres, F. Aumayr, D. Carralero, L. Guimarãis, and B. Kurzan, Plasma Phys. Controlled Fusion 56, 025008 (2014).

[23] S. P. Hirshman et al., Phys. Fluids 26, 3553 (1983).

[24] E. Strumberger, S. Günter, and C. Tichmann, Nucl. Fusion 54, 064019 (2014).

[25] J. Loizu, S. R. Hudson, P. Helander, S. A. Lazerson, and A. Bhattacharjee, Phys. Plasmas 23, 055703 (2016).

[26] S. A. Lazerson, J. Loizu, S. Hirshman, and S. R. Hudson, Phys. Plasmas 23, 012507 (2016).

[27] S. K. Rathgeber, L. Barrera, T. Eich, R. Fischer, B. Nold, W. Suttrop, M. Willensdorfer, and E. Wolfrum, Plasma Phys. Controlled Fusion 55, 025004 (2013).

[28] J. A. Morales et al., Phys. Plasmas 23, 042513 (2016).

[29] W. Park, E. D. Fredrickson, A. Janos, J. Manickam, and W. M. Tang, Phys. Rev. Lett. 75, 1763 (1995).

[30] C. C. Hegna, Phys. Plasmas 7, 3921 (2000).

[31] T. B. Cote et al. (to be published).

[32] A. Kendl, and H. Wobig, Phys. Plasmas 6, 4714 (1999).

[33] G. Birkenmeier, M. Ramisch, G. Fuchert, A. Köhn, B. Nold, and U. Stroth, Plasma Phys. Controlled Fusion 55, 015003 (2013).

[34] T. Weyens, R. Sánchez, G. Huijsmans, A. Loarte, and L. García, J. Comput. Phys. 330, 997 (2017).

[35] I. Holod, Z. Lin, S. Taimourzadeh, R. Nazikian, D. Spong, and A. Wingen, Nucl. Fusion 57, 016005 (2017).

[36] M. Becoulet et al., Phys. Rev. Lett. 113, 115001 (2014). 